\title{
Percepción sobre la responsabilidad social corporativa de los estudiantes de educación superior de Chile
}

\author{
Pedro Severino-González ${ }^{1}$, Jose Villalobos-Antunez ${ }^{2,3}$, Javiera Vergara-Gómez ${ }^{3}$ y María Yáñez-Venegas ${ }^{4}$ \\ (1) Facultad de Ciencias Sociales y Económicas, Departamento de Economía y Administración, Universidad Católica del \\ Maule, Talca, Chile (correo-e: pseverino@ucm.cl) \\ (2) Dirección de Investigación, Universidad Pedro de Valdivia, Santiago, Chile (correo-e: jvvillalobos@gmail.com) \\ (3) Facultad Experimental de Ciencias, Departamento de Ciencias Humanas, Universidad del Zulia, Maracaibo, \\ Venezuela (correo-e: jvvillalobos@gmail.com) \\ (4) Facultad de Ciencias Sociales y Económicas, Escuela de Ingeniería Comercial, Universidad Católica del Maule, \\ Talca, Chile (correo-e: vergarajaviera96@gmail.com; katalinayanez96@gmail.com)
}

Recibido Dic. 18, 2020; Aceptado Feb. 22, 2021; Versión final Mar. 28, 2021, Publicado Ago. 2021

\section{Resumen}

Esta investigación tiene como propósito explorar la percepción de los estudiantes de educación superior sobre la responsabilidad social corporativa. El estudio de la responsabilidad social corporativa es un tema de relevancia debido a las nuevas exigencias de los diversos stakeholders. Se utiliza un cuestionario cuantitativo, dando paso a la aplicación de análisis factorial exploratorio y diferencias de medias. Los resultados muestran diferencias significativas según género, edad y nivel de ingreso familiar. Las mujeres son las que presentan una mayor valoración en relación a las acciones filantrópicas desarrolladas por las empresas, dichas acciones filantrópicas son reconocidas por los estudiantes entre 18 a 24 años; todo lo anterior se encuentra en correspondencia con el nivel de ingreso familiar. Se concluye que es necesario que los estudiantes sean entrenados en responsabilidad social personal y social, como a su vez, en ética empresarial aplicada, lo que debería permitir el despliegue natural de procesos decisionales que procuren el bienestar de todos los grupos de interés.

\section{Perception of corporate social responsibility by higher education students in Chile}

\begin{abstract}
The purpose of this research study is to explore the perception about corporate social responsibility by higher education students. This a relevant topic due to the new demands from stakeholders. A quantitative questionnaire is used. Exploratory factor analyses and mean differences are applied. The results show significant differences according to gender, age, and family income level. Depending on family income level, women most value company-developed philanthropic initiatives and students, ages 18 to 24, acknowledge such initiatives. It is concluded that it is necessary to train students on social and personal responsibility and on applied corporate ethics to enhance natural decision-making processes aiming at improving the well-being of all groups of interest.
\end{abstract}




\section{INTRODUCCIÓN}

La responsabilidad social es un tema de investigación que ha sido abordado desde diversas perspectivas y disciplinarias, tales como: empresarial, universitaria, hospitalaria, sustentabilidad, entre otros (Padilla et al., 2017; Severino-González et al., 2020a). Ahora bien, las indagaciones sobre responsabilidad social empresarial (RSE) o responsabilidad social corporativa (RSC) -en la presente investigación se consideran ambos sin distinción alguna, reconociendo que existen algunas divergencias-, se concentran en estudios que exploran el comportamiento del consumidor (Arredondo Trapero et al., 2011; Gutiérrez-Rodríguez et al., 2017); siendo un grupo de interés que define la teoría de los stakeholder. Lo cual es fruto del dinamismo y la complejidad que tiene la aplicación de sus principios y valores éticos en la gestión organizacional (Stankowitz Penteado et al., 2013; Acuña-Moraga et al., 2020), las que buscan generar beneficios colectivos para todo el econsistema empresarial, pudiendo ser clasificadas en responsabilidades económicas, éticas, legales y filantrópicas (Carroll, 1999; Carroll y Shabana, 2010).

La RSC es dinámica ya que se configura, transforma y adapta según el contexto -características propias de los territorios- y, las revoluciones de los saberes; en éste último caso se encuentra la revolución de los valores (Stankowitz et al., 2013; Haski-Leventha et al., 2017). Estos cambios han transformado las expectativas, percepciones e ideales valóricos de los individuos sobre el actuar de las organizaciones (Severino-González et al., 2021). Frente a este escenario, las compañías han desarrollado diversas estrategias que vienen a atender las nuevas exigencias de los stakeholders, implementando programas, planes y modelos que demuestren su compromiso con la sociedad y el medioambiente (Cuervo-Cazurra, 2018; Kim, 2019). Por otro lado, los nuevos requerimientos de los diversos grupos de interés, han motivado a la implementación de iniciativas que contribuyen a la generación de mayores oportunidades para todos los intervinientes; lo que conduce al mejoramiento del bienestar y calidad de vida de la sociedad, las que deben ser coherentes, sustentables y pertinentes, los que no deben dejar de lado los valores individuales y sociales (Bénabou y Tirole, 2010; González-Gijón et al., 2020). Esta nueva conciencia de RSE y respeto al medioambiente, se fundamenta en criterios éticos e íntegros -y no cosméticos- que tienen como finalidad construir una sociedad más justa y sostenible (Severo et al., 2018).

Ahora bien, la importancia en la consideración de los educandos de nivel superior en correspondencia con la RSC, radica en el rol que desempeñan como estudiantes (Severino-González et al., 2019) y, como futuros profesionales (Martelo et al., 2018), los cuales serán interpelados a través de problemas organizacionales, dilemas éticos y cuestionamientos sociales; conduciéndolos a tomar decisiones (Eweje y Brunton, 2010; Severino-González et al., 2020b). Lo antes señalado, puede ser investigado a través de la propuesta de Carroll (1991), que revela el funcionamiento de las empresas desde la perceptiva de los estudiantes universitarios (Mazur y Walczyna, 2021). Lo que, además, debería justiciar la importancia de la enseñanza de la ética empresarial y la RSC (Rodríguez-Gómez et al., 2020). En este contexto, las aplicaciones teóricas y prácticas de la RSC pueden ayudar a gerentes, administradores y operarios a reducir las eventuales incongruencias en los negocios e, incentivarlos a hacer lo correcto y/o adecuado, contribuyendo al desarrollo y progreso de las organizaciones y, la comunidad en general, buscado siempre soluciones armónicas y equitativas para con la sociedad en general y, el medioambiente natural.

La RSC, según Carroll (1991) está compuesta por cuatro constructos: responsabilidad económica, responsabilidad legal, responsabilidad ética y responsabilidad filantrópica. Por su lado, la responsabilidad económica, se refiere a las pretensiones de la sociedad sobre la empresa como generadora de rentabilidad gracias a la fabricación de bienes valorados por los mismo. Ahora bien, la responsabilidad legal, son expectativas de funcionamiento de la compañía considerando en todo momento las disposiciones legales, lo que no se puede ante poner a los objetivos económicos. Por otro lado, la responsabilidad ética, está en correspondencia con el deseo de un comportamiento bajo normas éticas compartidas por la sociedad los cuales deben estar por sobre la exclusiva rentabilidad. Por último, la responsabilidad filantrópica, expectativas de la sociedad en cuanto al involucramiento a problemas sociales, asuntos públicos y, la disposición de recursos para fines filantrópicos (Carroll y Shabana, 2010). Lo antes mencionado ha permitido el diseño de instrumentos cuantitativos, los cuales han sido aplicado en diversos sujetos y contextos (Maignan, 2001; Wendlandt Amezaga et al., 2016).

Es por ello que, es relevante estudiar las percepciones de los futuros tomadores de decisión según características sociodemográficas (Larrán et al., 2018; Almutawa y Hewaidy, 2020), para lo cual se ha definido la siguiente pregunta de investigación ¿Cuáles son las percepciones de los estudiantes de educación superior sobre la RSC? Lo anterior, permite definir el propósito: explorar las percepciones de los estudiantes de educación superior sobre la responsabilidad social corporativa. En relación a las hipótesis, se planten las siguientes: hipótesis nula ( $\mathrm{H0})$ : No existen diferencias estadísticamente significativas según característica sociodemográfica sobre la percepción de los estudiantes sobre la RSC y, en cuanto a la hipótesis alterna $(\mathrm{H} 1)$ : Existen diferencias estadísticamente significativas según característica sociodemográfica sobre la 
percepción de los estudiantes sobre la RSC. Lo anterior, se logra sustentar gracias al desarrollo de un análisis factorial exploratorio y el examen de diferencias de medias estadísticamente significativas. Todo lo cual, contribuye a la toma de decisiones tanto para instituciones de educación superior, como así también para empresas de diversos sectores industriales (Pätäri et al., 2017; Ugwuozor, 2020).

Para facilitar la comprensión del texto, este manuscrito se estructura de la siguiente forma: en la primera sección, se exponen los antecedentes teóricos que presentan la relevancia de indagación; en la segunda sección, se exponen las decisiones metodológicas; en la tercera sección, se explican los principales hallazgos; en la cuarta sección se desarrolla la respectiva discusión y; finalmente, en la quinta sección se exhiben las conclusiones.

\section{METODOLOGÍA}

En esta sección se entregan los principales aspectos sobre las características de los participantes y las técnicas para el levantamiento de información, además se detallan cada uno de los procedimientos. Por otro lado, se explican los antecedentes del instrumento y, finalmente se señalan las estrategias de análisis en relación al propósito de esta investigación.

\section{Participantes}

Esta indagación considera la participación de 204 estudiantes de educación superior de una ciudad ubicada en la zona centro sur de Chile (tasa de repuesta=95,1\%). Para la obtención de la muestra se realizó un muestreo no probabilístico por conveniencia, lo cual es producto de la crisis social experimentada en octubre de 2019. En la tabla 1, se pueden observar las características de cada uno de los estudiantes. En cuanto al género la muestra se encuentra relativamente equilibrada. En relación a la edad la mayor concentración declara tener entre 18 a 24 años (87.25\%). En correspondencia con la ocupación, una elevada cantidad participantes declara solo estudiar (80.88\%). En su mayoría los estudiantes señalan tener entre 4 a 6 años de permanencia en las casas de estudios superiores (64.22\%). En relación a los miembros del grupo familiar, en su mayoría declara que lo integran entre 3 a 4 personas (57.84\%). En cuanto al nivel de ingreso, existe una alta dispersión, la mayor concentración señala tener como ingreso familiar entre $\$ 200.000$ (CL) y 600.00 (CL). La mayoría declara provenir de un territorio urbano (74.51\%). En su mayoría los estudiantes declaran no integrar un grupo de voluntariado (80.88\%). Finalmente, $62.75 \%$ de los estudiantes declaran no conocer el concepto de RSE.

Tabla 1: Características sociodemográficas de los participantes.

\begin{tabular}{|c|c|c|}
\hline Característica & Categoría & Porcentaje (\%) \\
\hline \multirow[t]{2}{*}{ Género } & Mujer & $57.35 \%$ \\
\hline & Hombre & $42.65 \%$ \\
\hline \multirow[t]{2}{*}{ Edad } & De 18 a 24 años & $87.25 \%$ \\
\hline & De 25 a 31 años & $12.75 \%$ \\
\hline \multirow[t]{2}{*}{ Ocupación } & Sólo estudia & $80.88 \%$ \\
\hline & Estudia y trabaja & $19.12 \%$ \\
\hline \multirow[t]{3}{*}{ Permanencia } & De 1 a 3 años & $29.41 \%$ \\
\hline & De 4 a 6 años & $64.22 \%$ \\
\hline & De 7 a 9 años & $6.37 \%$ \\
\hline \multirow[t]{3}{*}{ Grupo familiar } & De 1 a 2 integrantes & $12.75 \%$ \\
\hline & De 3 a 4 integrantes & $57.84 \%$ \\
\hline & De 5 a 6 integrantes & $29.41 \%$ \\
\hline \multirow[t]{6}{*}{ Nivel de ingreso familiar (CL) } & Menor a $\$ 200.000$ & $5.39 \%$ \\
\hline & $\$ 200.000-\$ 400.000$ & $26.96 \%$ \\
\hline & $\$ 400.001-\$ 600.000$ & $25.98 \%$ \\
\hline & $\$ 600.001-\$ 800.000$ & $16.67 \%$ \\
\hline & $\$ 800.001-\$ 1.000 .000$ & $8.82 \%$ \\
\hline & Mayor a $\$ 1.000 .000$ & $16.18 \%$ \\
\hline \multirow[t]{2}{*}{ Territorio } & Urbano & $74.51 \%$ \\
\hline & Rural & $25.49 \%$ \\
\hline \multirow[t]{2}{*}{ Voluntariado } & $\mathrm{Si}$ & $19.12 \%$ \\
\hline & No & $80.88 \%$ \\
\hline \multirow[t]{2}{*}{ Conoce RSE } & $\mathrm{Si}$ & $37.25 \%$ \\
\hline & No & $62.75 \%$ \\
\hline
\end{tabular}




\section{Procedimientos}

La recolección de datos se desarrolló de manera online entre agosto y diciembre de 2019, producto del estallido social y las movilizaciones sociales que se generaron durante los últimos meses del 2019 en Chile. En todo momento se explicó a los participantes la confidencialidad, anonimato y el carácter voluntario de ésta indagación. Una vez, levantadas las percepciones la información fue sistematizada en Microsoft Excel 2016, para ser exportada a SPSS18.

\section{Instrumento}

El instrumento utilizado fue diseñado por Maignan (2001) y aplicado en su versión en español por Wendlandt Amezaga et al., (2016), el cual analiza la percepción de las expectativas de los stakeholders, considerando como principio rector la teoría de la pirámide de Carroll (1991). En cuanto a la confiabilidad del instrumento, el estadístico de Alfa de Cronbach posee un coeficiente de 0.804 (Wendlandt Amezaga et al., 2016).

En relación a la estructura del instrumento, la primera parte posee preguntas filtro, que permiten asegurar que sean estudiantes de educación superior; la segunda sección, está integrada por preguntas sociodemográficas y; en la tercera sección, se presenta el cuestionario compuesto por 16 afirmaciones distribuidas en cuatro dimensiones que configuran el concepto de responsabilidad social empresarial (Carroll, 1991): 1. Responsabilidad Económica, 2. Responsabilidad Legal, 3. Responsabilidad Ética y 4. Responsabilidad Filantrópica (ver tabla 2). En cuanto al tipo de respuesta es a través de una escala de tipo Likert con seis opciones, las cuales son 1 = Muy en desacuerdo, 2 = En desacuerdo, $3=$ Ligeramente en desacuerdo, $4=$ Ligeramente de acuerdo, $5=$ De acuerdo y $6=$ Muy de acuerdo.

Tabla 2: Cuestionario de percepción de responsabilidad social corporativa

\begin{tabular}{|l|c|l|}
\hline Dimensión & Variable & \multicolumn{1}{|c|}{ Expectativas } \\
\hline \multirow{4}{*}{ Económica } & $\mathrm{v} 1$ & Maximizar las ganancias. \\
\cline { 2 - 4 } & $\mathrm{v} 2$ & Controlar estrictamente sus costos de producción. \\
\cline { 2 - 4 } & $\mathrm{v} 3$ & Planear el éxito a largo plazo. \\
\cline { 2 - 4 } & $\mathrm{v} 4$ & Mejorar siempre los resultados económicos. \\
\hline \multirow{4}{*}{ Legal } & $\mathrm{v} 5$ & $\begin{array}{l}\text { Asegurarse de que sus empleados actúan dentro de los estándares definidos por la } \\
\text { ley. }\end{array}$ \\
\cline { 2 - 4 } & $\mathrm{v} 6$ & Cumplir con sus obligaciones contractuales. \\
\cline { 2 - 4 } & $\mathrm{v} 7$ & Evitar infringir la ley, incluso si esto ayuda a mejorar el rendimiento. \\
\cline { 2 - 4 } & $\mathrm{v} 8$ & Respetar siempre los principios definidos por el sistema regulatorio. \\
\hline \multirow{5}{*}{ Ética } & $\mathrm{v} 9$ & $\begin{array}{l}\text { Cumplir con los aspectos éticos aunque afecten negativamente el desempeño } \\
\text { económico. }\end{array}$ \\
\cline { 2 - 4 } & $\mathrm{v} 10$ & $\begin{array}{l}\text { Asegurar que el respeto a los principios éticos tiene prioridad sobre el desempeño } \\
\text { económico }\end{array}$ \\
\cline { 2 - 4 } & $\mathrm{v} 11$ & Estar comprometidas con principios éticos bien definidos. \\
\cline { 2 - 4 } & $\mathrm{v} 12$ & Evitar comprometer los estándares éticos a fin de lograr los objetivos corporativos. \\
\hline & $\mathrm{v} 13$ & Ayudar a resolver problemas sociales. \\
\cline { 2 - 3 } & $\mathrm{v} 14$ & Participar en la gestión de los asuntos públicos. \\
\cline { 2 - 3 } & $\mathrm{v} 15$ & Destinar parte de sus recursos a las actividades filantrópicas. \\
\cline { 2 - 3 } & $\mathrm{v} 16$ & $\begin{array}{l}\text { Desempeñar un papel importante en nuestra sociedad más allá de la pura } \\
\text { generación de beneficios. }\end{array}$ \\
\hline
\end{tabular}

\section{Estrategias de análisis}

En SPSS18 se efectuaron todos los análisis estadísticos, por ejemplo: medias, desviaciones estándar, coeficientes de consistencia interna y correlaciones entre las variables. Ahora bien, previamente se desplegó un análisis factorial exploratorio, se aplicó el método de extracción de componentes principales y rotación Varimax (Lloret-Segura et al., 2014). Luego, se aplicaron pruebas de normalidad y test estadísticos para la exploración de diferencias de medias estadísticamente significativas.

\section{RESULTADOS}

En esta sección se entregan los hallazgos más relevantes de esta indagación. En primer lugar, se desarrolla un análisis factorial exploratorio, posteriormente se calculan algunos estadísticos descriptivos y, de consistencia interna. Finalmente, se exploran las diferencias estadísticamente significativas. 


\section{Análisis factorial exploratorio}

Para el análisis factorial exploratorio se determinaron algunos indicadores que permiten establecer la pertinencia del mismo. El Kaiser-Meyer-Olkin (KMO) es 0.919, la prueba de esfericidad de Bartlett es 0.000 , con $\mathrm{gl}=120$. Todo lo anterior, entrega las credenciales para continuar con la aplicación del mismo. Se aplicó el método de extracción de componentes principales y la rotación Varimax. Las dimensiones se agrupan de la siguiente manera: Responsabilidad Legal: v6, v5, v7 y v8, Responsabilidad Filantrópica: v14, v15, v13 y v16, Responsabilidad Económica: v1, v4, v2, v3 y, Responsabilidad Ética: v12, v10, v9 y v11 (ver tabla 3).

Tabla 3: Matriz de factores rotados de la responsabilidad social corporativa

\begin{tabular}{|c|c|c|c|c|}
\hline \multirow[b]{2}{*}{ Variable } & \multicolumn{4}{|c|}{ Dimensiones } \\
\hline & $\begin{array}{c}\text { 1. Responsabilidad } \\
\text { Legal }\end{array}$ & $\begin{array}{l}\text { 2. Responsabilidad } \\
\text { Filantrópica }\end{array}$ & $\begin{array}{l}\text { 3. Responsabilidad } \\
\text { Económico }\end{array}$ & $\begin{array}{c}\text { 4. Responsabilidad } \\
\text { Ética }\end{array}$ \\
\hline v6 & 0.784 & & & \\
\hline v5 & 0.765 & & & \\
\hline v7 & 0.744 & & & \\
\hline v8 & 0.739 & & & \\
\hline v14 & & 0.834 & & \\
\hline v15 & & 0.762 & & \\
\hline $\mathrm{v} 13$ & & 0.76 & & \\
\hline v16 & & 0.639 & & \\
\hline v1 & & & 0.796 & \\
\hline v4 & & & 0.769 & \\
\hline v2 & & & 0.727 & \\
\hline v3 & & & 0.675 & \\
\hline v12 & & & & 0.743 \\
\hline v10 & & & & 0.709 \\
\hline v9 & & & & 0.675 \\
\hline v11 & & & & 0.650 \\
\hline
\end{tabular}

Ahora bien, una vez desarrollado el análisis factorial exploratorio, se efectuaron análisis de consistencia interna y se determinaron medias, desviaciones estándar y correlaciones (ver tabla 4). Como se puede observar en cada uno de los casos, los coeficientes de consistencia interna son superiores a 0.8; lo que es considerado satisfactorio y muy alto. En relación a la media, el mayor valor se encuentra en la dimensión Responsabilidad Legal (Media=5.417), seguido de cerca por la dimensión Responsabilidad Ética (Media=5.191). Ahora bien, en cuanto a la asociación entre las dimensiones, se aplicó el test Spearman Rho debido a la distribución no normal de los datos, en este caso la mayor relación se encuentra entre las dimensiones Responsabilidad Legal y Responsabilidad Ética $(\rho=0.52)$.

Tabla 4: Estadísticos descriptivos, niveles de consistencia interna por dimensión y correlaciones

\begin{tabular}{|l|c|c|c|c|c|c|c|}
\hline \multicolumn{1}{|c|}{ Dimensiones } & Media & $\begin{array}{c}\text { Desviación estándar } \\
(D E)\end{array}$ & $\begin{array}{c}\text { Alfa de } \\
\text { Cronbach }\end{array}$ & 1 & 2 & 3 & 4 \\
\hline 1. Responsabilidad Legal & 5.417 & 0.03 & 0.905 & 1 & & & \\
\hline 2. Responsabilidad Filantrópica & 4.963 & 0.27 & 0.863 & 0.38 & 1 & & \\
\hline 3. Responsabilidad Económica & 4.789 & 0.16 & 0.803 & 0.22 & 0.28 & 1 & \\
\hline 4. Responsabilidad Ética & 5.191 & 0.19 & 0.869 & 0.52 & 0.39 & 0.21 & 1 \\
\hline
\end{tabular}

\section{Diferencias de medias}

Es preciso señalar que, para cada una de las estimaciones de diferencias de medias, se aplicaron pruebas de normalidad, determinando según el indicador Kolmogorov-Smirnov que, en todos los casos los datos responden a una distribución no normal. Se exploraron diferencias estadísticamente significativas según género, edad, ocupación, voluntariado, nivel de ingresos familiar, integrantes grupo familiar, territorio, permanencia y conocimiento de RSE. Ahora bien, solo se encuentran diferencias significativas, según: género, edad y nivel de ingreso familiar; en este sentido es preciso indicar que, según edad y nivel de ingreso familiar no poseen una distribución equilibrada de participantes. A continuación, se entregan detalles al respecto. 
En la tabla 5, se puede observar diferencias de medias estadísticamente significativas según prueba no paramétrica $U$ de Mann-Whitney en la dimensión Responsabilidad Filantrópica ( $p$-valor=0.029). En éste caso las mujeres son las que presentan una mayor valoración (Media=4.91; $\mathrm{DE}=1.11$ ), lo anterior se encuentra en correspondencia con el rol que poseen las empresas frente a la sociedad, lo que implica el compromiso de la compañía por problemas sociales, asuntos públicos, entre otros; todo lo cual considera las diversas necesidades que subyacen del tejido social.

Tabla 5: Media, desviaciones estándar y diferencias según género

\begin{tabular}{|c|c|c|c|c|}
\hline Dimensiones & Género & Media & Desviación Estándar & $p$-valor \\
\hline \multirow{2}{*}{ 1. Responsabilidad Legal } & Mujer & 5.42 & 1.01 & \multirow{2}{*}{0.723} \\
\hline & Hombre & 5.42 & 1.00 & \\
\hline \multirow{2}{*}{ 2. Responsabilidad Filantrópica } & Mujer & 4.91 & 1.11 & \multirow{2}{*}{0.029} \\
\hline & Hombre & 4.89 & 1.12 & \\
\hline \multirow{2}{*}{ 3. Responsabilidad Económica } & Mujer & 4.78 & 1.16 & \multirow{2}{*}{0.809} \\
\hline & Hombre & 4.82 & 1.16 & \\
\hline \multirow{2}{*}{ 4. Responsabilidad Ética } & Mujer & 5.23 & 1.07 & \multirow{2}{*}{0.873} \\
\hline & Hombre & 5.19 & 1.11 & \\
\hline
\end{tabular}

En la tabla 6 , se puede observar diferencias estadísticamente significativas en relación a la edad en las dimensiones Responsabilidad Filantrópica ( $p$-valor $=0.027$ ) y Responsabilidad Ética ( $p$-valor=0.037), según la prueba no paramétrica $U$ de Mann-Whitney. En cuanto a la dimensión Responsabilidad Filantrópica, los estudiantes que tienen entre 18 a 24 años, presentan una mayor valoración (Media=5.32; DE=1.12), debido a que consideran que las compañías deben cumplir con sus responsabilidades ligadas al altruismo y la solidaridad, las que subyacen de la comunidad y ameritan el involucramiento de las empresas. Por otro lado, en relación a la dimensión Responsabilidad Ética, los estudiantes que tienen entre 18 a 24 años presentan una mayor valoración (Media=5.11; $\mathrm{DE}=1.23$ ), en éste sentido las empresas deben resguardar y respetar principios éticos, por sobre principios económicos y objetivos corporativos.

Tabla 6: Media, desviaciones estándar y diferencias según edad

\begin{tabular}{|c|c|c|c|c|}
\hline Dimensiones & Edad & Media & Desviación Estándar & $p$-valor \\
\hline \multirow{2}{*}{ 1. Responsabilidad Legal } & De 18 a 24 años & 5.32 & 1.12 & \multirow{2}{*}{0.259} \\
\hline & De 25 a 31 años & 5.28 & 1.19 & \\
\hline \multirow{2}{*}{ 2. Responsabilidad Filantrópica } & De 18 a 24 años & 4.81 & 1.19 & \multirow{2}{*}{0.027} \\
\hline & De 25 a 31 años & 4.75 & 1.26 & \\
\hline \multirow{2}{*}{ 3. Responsabilidad Económica } & De 18 a 24 años & 4.65 & 1.23 & \multirow{2}{*}{0.100} \\
\hline & De 25 a 31 años & 4.62 & 1.25 & \\
\hline \multirow{2}{*}{ 4. Responsabilidad Ética } & De 18 a 24 años & 5.11 & 1.23 & \multirow{2}{*}{0.037} \\
\hline & De 25 a 31 años & 5.01 & 1.35 & \\
\hline
\end{tabular}

En la tabla 7, se puede observar que exclusivamente en la dimensión Responsabilidad Filantrópica, presenta diferencias de medias estadísticamente significativas según la prueba no paramétrica de Kruskal-Wallis en correspondencia con el ingreso familiar. En éste caso, se puede señalar que los estudiantes que indican tener un ingreso familiar entre $\$ 800.001-\$ 1.000 .000$ (CL), declaran leventemente una mayor expectativa (Media=4.89; $\mathrm{DE}=1.13$ ), lo que tiene relación con las responsabilidades emanadas producto del involucramiento para la resolución de problemas sociales, asuntos pública y actividades generosas. Ahora bien, aunque las medias entre cada uno de los subgrupos son muy similares las percepciones entre subgrupos difieren significativamente según prueba antes señalada.

Tabla 7. Media, desviaciones estándar y diferencias según nivel de ingreso familiar

\begin{tabular}{|c|c|c|c|c|}
\hline Dimensiones & Nivel de ingreso familiar (CL) & Media & Desviación Estándar & $p$-valor \\
\hline \multirow[t]{6}{*}{ 1. Responsabilidad Legal } & Menor a $\$ 200.000$ & 5.31 & 1.04 & \multirow[t]{6}{*}{0.068} \\
\hline & $\$ 200.000-\$ 400.000$ & 5.39 & 0.99 & \\
\hline & $\$ 400.001-\$ 600.000$ & 5.38 & 1.00 & \\
\hline & $\$ 600.001-\$ 800.000$ & 5.40 & 0.99 & \\
\hline & $\$ 800.001-\$ 1.000 .000$ & 5.40 & 0.96 & \\
\hline & Mayor a $\$ 1.000 .000$ & 5.38 & 0.99 & \\
\hline \multirow[t]{6}{*}{ 2. Responsabilidad Filantrópica } & Menor a $\$ 200.000$ & 4.84 & 1.17 & \multirow[t]{6}{*}{0.028} \\
\hline & $\$ 200.000-\$ 400.000$ & 4.88 & 1.14 & \\
\hline & $\$ 400.001-\$ 600.000$ & 4.85 & 1.15 & \\
\hline & $\$ 600.001-\$ 800.000$ & 4.88 & 1.13 & \\
\hline & $\$ 800.001-\$ 1.000 .000$ & 4.89 & 1.13 & \\
\hline & Mayor a $\$ 1.000 .000$ & 4.87 & 1.16 & \\
\hline
\end{tabular}


Tabla 7: continuación.

\begin{tabular}{|c|l|c|c|c|}
\hline Dimensiones & Nivel de ingreso familiar (CL) & Media & Desviación Estándar & $p$-valor \\
\hline 3. Responsabilidad Económica & Menor a $\$ 200.000$ & 4.70 & 1.20 & 0.834 \\
\cline { 2 - 5 } & $\$ 200.000-\$ 400.000$ & 4.76 & 1.16 \\
\cline { 2 - 5 } & $\$ 400.001-\$ 600.000$ & 4.78 & 1.15 \\
\cline { 2 - 5 } & $\$ 600.001-\$ 800.000$ & 4.79 & 1.16 \\
\cline { 2 - 5 } & $\$ 800.001-\$ 1.000 .000$ & 4.80 & 1.16 \\
\cline { 2 - 5 } & Mayor a $\$ 1.000 .000$ & 4.80 & 1.18 & \\
\hline 4. Responsabilidad Ética & Menor a $\$ 200.000$ & 5.17 & 1.07 & 1.05 \\
\cline { 2 - 5 } & $\$ 200.000-\$ 400.000$ & 5.19 & 1.07 \\
\cline { 2 - 5 } & $\$ 400.001-\$ 600.000$ & 5.18 & 1.06 \\
\cline { 2 - 5 } & $\$ 600.001-\$ 800.000$ & 5.15 & 1.10 \\
\cline { 2 - 5 } & $\$ 800.001-\$ 1.000 .000$ & 5.11 & 1.14 \\
\cline { 2 - 5 } & Mayor a $\$ 1.000 .000$ & & \\
\end{tabular}

\section{DISCUSIÓN}

El presente artículo declara como propósito explorar la percepción de los estudiantes de educación superior sobre la responsabilidad social corporativa, para lo cual se aplicó un instrumento cuantitativo a una muestra no probabilística. En cuanto a los resultados, solo en algunos casos en relación a las variables sociodemográficas se encontraron diferencias estadísticamente significativas. Ahora bien, a continuación, se tensionan los resultados con los hallazgos encontrados en la literatura. Según Pätäri et al. (2017), no se encuentran diferencias estadísticamente significas en su estudio sobre percepciones de los estudiantes como futuros consumidores con respecto a la RSC y la industria forestal. En cambio, en esta indagación que levanta la percepción de los estudiantes sobre la RSC sin considerar particularmente una industria, se encuentran diferencias estadísticamente significativas en género, edad y nivel de ingreso familiar. Algunas de ellas, también se encuentran en Larrán et al. (2018), Ugwuozor (2020) y, Almutawa y Hewaidy (2020), las que a continuación se colocan en tensión.

Por otro lado, en relación al género en esta indagación se presentan diferencias estadísticamente significativas en la dimensión Responsabilidad Filantrópica. En cambio, en la investigación de Almutawa y Hewaidy (2020), se encuentran diferencias significativas exclusivamente en la dimensión Responsabilidad Económicas. En el primer caso, la mayor valoración de las mujeres, es debido al reconocimiento del altruismo y solidaridad que deben considerar las compañías en sus gestiones. En cambio, según Almutawa y Hewaidy (2020), los hombres expresan una mayor valoración en prácticas que contribuyen a la ganancia de la compañía y, éste, a su vez, como consecuencia del éxito y la posición competitiva.

En esta indagación, se encuentran diferencias estadísticamente significativas en correspondencia con la edad de los estudiantes, lo que permite inferir en relación a los años de permanencia de los educandos en las casas de estudios superiores. Diferencias que, según Ugwuozor (2020), también son encontradas tras el desarrollo de estrategias de educación en ética empresarial. Ahora bien, Larrán et al. (2018), señala que los estudiantes de primeros años de carrera presentan una mayor preocupación en temas relacionados con la RSE en la sociedad, lo que coincide con los hallazgos en de ésta investigación en todas las dimensiones de la RSE. Lo que puede ser producto del desconocimiento sobre las implicaciones de la implementación de prácticas de responsabilidad social corporativa y, a su vez, el impacto que tienen en las finanzas de las compañías. A su vez, podría estar en correspondencia por el sentido de urgencia que tiene la RSC en relación a la contribución que genera al bienestar de las familias de los estudiantes y, a ellos mismos, a través de diversas asistencias y ayudas expresadas en recursos económicos y no económicos.

Por otro lado, se encuentran diferencias estadísticamente significativas en la dimensión responsabilidad Filantrópica, debido a la valoración otorgada por los educandos en correspondencia a las prácticas asociadas a la ayuda a problemas sociales, participación en políticas públicas, además de las donaciones y, el ecuánime esfuerzo adicional a favor de la comunidad. Lo antes señalado, se encuentra en armonía con Ortega-Vivanco et al. (2021), entre sus hallazgos los estudiantes valoran a las empresas que dedican esfuerzos para aportar a la solución de problemas sociales y ambientales, además de la promoción de los derechos humanos, erradicación de la discriminación y el apoyo a causas solidarias.

Los hallazgos de esta indagación entregan antecedentes que pueden ser utilizadas para la toma de decisiones tanto por instituciones que se dedican a la formación de profesionales y, también, por organizaciones públicas y privadas. Por un lado, las instituciones de educación superior, pueden a través de estos resultados manifestar el sentido de urgencia que tiene la incorporación de políticas educativas que estimulen la conciencia social y, a su vez, los valores como la empatía, solidaridad, trasparencia y altruismo (Osuna y Luna, 2011); debido a que los estudiantes de hoy, serán los futuros tomadores de decisiones en las 
organizaciones y, por ende, eventuales procuradores de estrategias de RSC. Por otro lado, las organizaciones públicas y privadas, pueden hacer uso de estos revelamientos, para el desarrollo de estrategias focalizadas que beneficien a diversos grupos de interés según sus características sociodemográficas; en relación a las priorizaciones estratégicas según diversos criterios de clasificación; generando beneficios al econsistema empresarial.

\section{CONCLUSIONES}

Considerando el trabajo desarrollado y los principales hallazgos, se presentan las siguientes conclusiones:

1.- Es necesario que los estudiantes pueden ser entrenados en responsabilidad social personal y social, como a su vez, en ética empresarial aplicada; lo que debería permitir el despliegue natural de procesos decisionales que procuren el bienestar de todos los grupos de interés, basados en valores como la empatía, solidaridad, trasparencia y altruismo.

2.- Los resultados de esta indagación pueden ser utilizados por casas de estudios superiores y, las empresas públicas y privadas, para la generación de entornos pedagógicos y estratégicos que propicien el desarrollo de un comportamiento ético y socialmente responsable; todo lo cual, se ajusta a las prácticas de RSC. Las cuales pueden ser implementadas según las características sociodemográficas de los grupos de interés que forman parta del mapa estratégico de las organizaciones.

3.- Las futuras investigaciones tienen el desafío de aumentar el número de participantes, como, además, la exploración de diversos grupos de interés, permitiendo una mayor compresión del fenómeno social; motivando a la explicación de las casusas que caracterizan cada una de las percepciones sobre la RSE, lo que admite la consulta a futuros empleadores, políticos, dirigentes sociales, entre otros.

4.- Es importante que futuras investigaciones pueden tensionar los resultados de esta indagación en contraste con hallazgos post crisis sanitaria, para la exploración de brechas y, sus respectivas implicancias. Lo que puede conducir a la reconsideración de valores sociales e individuales, producto del relevamiento de lo transcendental y, la resignificación de la vida y las relaciones interpersonales.

\section{REFERENCIAS}

Acuña-Moraga, O., Severino-González, P., Garrido-Véliz, V., y Martin-Fiorino, V., Consumo sustentable y responsabilidad social. Una visión convergente que contribuye al desarrollo sustentable. Revista Interciencia, 45(8), 384389 (2020).

Almutawa, A., y Hewaidy, A.M., Accounting students' perception of corporate social responsibility: evidence from Kuwait. International Journal of Innovation, Creativity and Change, 14(9), 334- 356 (2020).

Arredondo Trapero, F.G., Maldonado De Lozada, V., y De la Garza García, J., El consumidor ante la responsabilidad social corporativa. Actitudes según edad y género. Cuadernos de Administración, 24(43), 285-305 (2011).

Bénabou, R., y Tirole, J., Individual and corporate social responsibility, https://doi.org/10.1111/j.14680335.2009.00843.x., Economica, 77(305), 1-19 (2010).

Carroll, A.B., y Shabana, K.M., The business case for corporate social responsibility: a review of concepts, research and practice, https://doi.org/10.1111/j.1468-2370.2009.00275.x, International Journal of Management Reviews, 12(1), 85105 (2010).

Carroll, A.B., Corporate social responsibility: evolution of a definitional construct, https://doi.org/10.1177/000765039903800303, Business \& society, 38(3), 268-295 (1999).

Carroll, A.B., The pyramid of corporate social responsibility: toward the moral management of organizational stakeholders, https://doi.org/10.1016/0007-6813(91)90005-G, Business Horizons, 34(4), 39-48 (1991).

Cuervo-Cazurra, A., The evolution of business groups' corporate social responsibility, https://doi.org/10.1007/s10551018-3912-4, Journal of Business Ethics, 153(4), 997-1016 (2018).

Eweje, G., y Brunton, M., Ethical perceptions of business students in a New Zealand university: do gender, age and work experience matter?, https://doi.org/10.1111/j.1467-8608.2009.01581.x, Business Ethics: A European Review, 19(1), 95111 (2010).

González-Gijón, G., Martínez-Heredia, N., Amaro-Agudo, A., y Soriano-Díaz, A., Estudio de los valores en el alumnado que cursa el grado de maestro en educación primaria en las universidades públicas de Andalucía, http://dx.doi.org/10.4067/S0718-50062020000200083, Formación universitaria, 13(2), 83-92 (2020).

Gutiérrez-Rodríguez, P., Cuesta-Valiño, P., y Vázquez-Burguete, J.L., The effects of corporate social responsibility on customer-based brand equity: Spanish hypermarket case, https://doi.org/10.1080/1331677X.2017.1305797, Economic Research-Ekonomska Istrazivanja, 30(1), 290-301 (2017). 
Haski-Leventhal, D., Pournader, M., y McKinnon, A., The role of gender and age in business students' values, CSR attitudes, and responsible management education: learnings from the PRME international survey, https://doi.org/10.1007/s10551-015-2936-2, Journal of Business Ethics, 146(1), 219-239 (2017).

Larrán, M., Andrades, J., y Herrera, J., An examination of attitudes and perceptions of Spanish business and accounting students toward corporate social responsibility and sustainability themes, https://doi.org/10.1016/j.rcsar.2018.02.001, Revista de Contabilidad-Spanish Accounting Review, 21(2), 196-205 (2018).

Lloret-Segura, S., Ferreres-Traver, A., Hernández-Baeza, A., y Tomás-Marco, I., El análisis factorial exploratorio de los ítems: una guía práctica, revisada y actualizada, https://doi.org/10.6018/analesps.30.3.199361, Anales de Psicología, 30(3), 1151-1169 (2014).

Maignan, I., Consumers' Perceptions of corporate social responsibilities: a cross-cultural comparison, https://doi.org/10.1023/A:1006433928640, Journal of Business Ethics, 30(1), 57-72 (2001).

Martelo, R.J., Jiménez-Pitre, I., y Quintana, A., Determinación del perfil profesional de estudiantes de pregrado aplicando la técnica de análisis comparativo, https://doi.org/10.4067/S0718-07642018000200029, Información tecnológica, 29(2), 29-40 (2018).

Mazur, B., y Walczyna, A., Corporate Social Responsibility in the Opinion of Polish and Foreign Students in Management Program of Lublin University of Technology, https://doi.org/10.3390/su13010333, Sustainability, 13(1), 333 (2021).

Ortega-Vivanco, M., Jaramillo-Loaiza, G., y Tandazo-Arias, T., Comportamiento de Compra y Responsabilidad Social de los Estudiantes Universitarios en Tiempos de COVID19: Ecuador, https://doi.org/10.19044/esj.2021.v17n4p80, European Scientific Journal, 17(4), 80 (2021).

Osuna, C., y Luna, E., Valores éticos en la formación universitaria de las áreas de ciencias naturales e ingeniería y tecnología, en el contexto de la sociedad del conocimiento, http://dx.doi.org/10.4067/S0718-50062011000500005, Formación universitaria, 4(5), 29-36 (2011).

Padilla, C., Arévalo, D., Bustamante, M., y Vidal, C. Responsabilidad social empresarial y desempeño financiero en la industria del plástico en Ecuador, https://doi.org/10.4067/S0718-07642017000400012, Información tecnológica, 28(4), 93-102 (2017).

Pätäri, S., Arminen, H., y otros 3 autores, Student values and perceptions of corporate social responsibility in the forest industry on the road to a bioeconomy, https://doi.org/10.1016/j.forpol.2017.10.009, Forest Policy and Economics, 85, 201-215 (2017).

Rodríguez-Gómez, S., Garde-Sánchez, R., Arco-Castro, M.L., y López-Pérez, M.V., Does the Use of Social Media Tools in Classrooms Increase Student Commitment to Corporate Social Responsibility?, https://doi.org/10.3389/fpsyg.2020.589250, Frontiers in Psychology, 11, 3690. (2020).

Severino-González, P., Acuña-Moraga, O., Astete-Tapia, K., y Medel-Maturana, J., Responsabilidad social y consumo sustentable. El caso de estudiantes de formación superior de Talca (Chile), http://dx.doi.org/10.4067/S071807642021000100143, Revista Información Tecnológica, 32(1), 143-150 (2021).

Severino-González, P., Villalobos Antúnez, J., González-Soto, N., y Ramírez Molina, R., Perspectiva de la responsabilidad social empresarial de la salud pública chilena, https://doi.org/10.37960/rvg.v25i92.34270, Revista Venezolana de Gerencia, 25(92), 1397-1421 (2020b).

Severino-González, P., Villalobos Antúnez, J., Romero-Argueta, J., y Garrido Véliz, V., Social responsibility of higher education students. Motivations for its development in times of Covid-19 in Chile and El Salvador, http://doi.org/10.5281/zenodo.4009788, Revista Utopía y Praxis Latinoamericana, 25(7), 439-452 (2020a).

Severino-González, P., Villalobos-Antúnez, J.V., y otros 3 autores, Social responsibility of university student and institutional educational policies. Recognizing the commitments to society. Revista Opción, 35(90), 1171-1197 (2019).

Severo, E.A., de Guimarães, J.C.F., y Dorion, E.C., Cleaner production, social responsibility and eco-innovation: generations' perception for a sustainable future, https://doi.org/10.1016/j.jclepro.2018.03.129, Journal of Cleaner Production, 186, 91-103 (2018).

Stankowitz Penteado, R.F., Mendes da Luz, L., y otros tres autores, Percepción de los estudiantes de ingeniería: tecnología y curso técnico sobre responsabilidad social empresarial, https://doi.org/10.4067/S071827242013000300012, Journal of technology management \& innovation, 8(1), 135-146 (2013).

Ugwuozor, F.O., Students' perception of corporate social responsibility: analyzing the influence of gender, academic status, and exposure to business ethics education, https://doi.org/10.1111/beer.12306, Business Ethics: A European Review, 29(4), 737-747 (2020).

Wendlandt Amezaga, T., Álvarez Medina, M., Nuñez Ramírez, M., y Valdez Pineda, D., Validación de un instrumento para medir la responsabilidad social empresarial en consumidores de México, https://doi.org/10.17230/ad-minister.29.4, AD-minister, (29), 79-100 (2016). 
